\title{
Central nervous system involvement in Sjögren's syndrome
}

\section{F C Soliotis, C P Mavragani, H M Moutsopoulos}

\section{Still an unresolved issue-multicentre studies are needed}

S jögren's syndrome (SS) is a relatively common autoimmune disorder, affecting $2-3 \%$ of the adult population. It is characterised by lymphocytic infiltration and destruction of the exocrine glands. The salivary and lachrymal glands are primarily affected, leading to a dry mouth and dry eyes, the hallmark of the disease. Other exocrine glands, which may be affected, include those of the pancreas, bronchial tree, and gastrointestinal tract. SS may occur alone (primary) or in association with other connective tissue diseases (secondary). The spectrum of clinical manifestations of SS is wide, ranging from mucosal dryness, directly related to exocrine dysfunction, to more systemic complaints, affecting mainly the musculoskeletal, pulmonary, renal, and vascular systems. ${ }^{1}$ Peripheral nervous system disease, manifested commonly as peripheral sensory neuropathy or more rarely as mononeuritis multiplex, is a well established feature of the disease, occurring in approximately a quarter of patients. ${ }^{2}$ However, involvement of the central nervous system (CNS) in patients with SS is a matter of serious controversy between different research groups, with a prevalence ranging from "undetectable" to "quite common". This discrepancy was previously attributed possibly to differences in the diagnostic criteria, selection of populations, special interests of different teams of investigators, or referral bias. $^{34}$

\section{PREVALENCE OF CNS DISEASE IN SS}

The prevalence of CNS disease in SS is a controversial topic. Tables 1 and 2 list the prevalence of CNS manifestations in patients with SS reported since 1985 The numbers quoted in publications range between 0 and $100 \% .^{25-21}$

Why is there is such a huge variability in the reported prevalence of CNS disease in SS?

Firstly, there is no consensus on the definition of CNS involvement. Some authors include psychiatric disease, but some do not. Also there is no consensus on the inclusion of mild symptoms such as headache or mood disturbances. Secondly, the diagnostic criteria used for SS are not uniform. Some authors have used stringent criteria that include confirmatory salivary gland biopsies, whereas others have included patients with probable SS. Thirdly, some studies have included patients with secondary SS, some of whom may have lupus with CNS disease. Also some patients may have true SS-systemic lupus erythematosus (SLE) overlap with CNS disease. Fourthly, confounding factors that may increase the risk for cerebrovascular disease such as diabetes, hypertension, and hyperlipidaemia or factors that may be associated with psychiatric disease such as thyroid disease are not always accounted for. This is important considering that the incidence of autoimmune endocrinopathies such as thyroid disease is increased in patients with SS. ${ }^{22}{ }^{23}$

The most important factor is the selection of patients studied and the presence of referral bias. Referral bias may occur in tertiary centres, where complex cases with severe disease are referred. This may lead to overdiagnosis of CNS disease as these patients may not represent the general SS population. Similarly, the prevalence of CNS disease would vary between patients seen in a neurology department and those seen in a rheumatology outpatient department and might lead to differences in disease prevalence quoted because of selection bias. Underdiagnosis may be a result of symptoms being dismissed by the assessing physician as functional or stress related. Patients may also fail voluntarily to report symptoms. This is of particular relevance for psychiatric symptoms. Furthermore, neurological complications in elderly patients with SS may be attributed to their age, again resulting in underdiagnosis. Furthermore, owing to a problem with classification, some patients with SS and CNS involvement may also satisfy the American College of Rheumatology criteria for SLE and hence may be diagnosed as CNS-SLE and therefore missed.

Finally, the reported prevalence of CNS disease may reflect true differences of coexistent CNS diseases in different populations due to different genetic and environmental factors. This is of particular relevance for diseases like multiple sclerosis (MS). The incidence of MS increases with latitude, and therefore coexistence of SS with MS may explain the high incidence of MS-like disease reported in North America and Scandinavia compared with the low incidence in south European countries and Colombia. ${ }^{24-26}$

To solve the controversy, prospective, controlled studies are needed with large numbers of patients, because the prevalence of specific neurological syndromes in the general population is low.

\section{AETIOLOGY: PATHOGENESIS}

The aetiology of CNS-SS involvement is not well delineated. However, some clues for its pathogenesis could be provided by cerebrospinal fluid (CSF) analysis, histopathology, and neuroimaging studies.

Some evidence points to an immunologically mediated mechanism of CNS disease in SS. Alexander et al examined the CSF of patients with SS with active CNS disease and found evidence of lymphocytosis, raised IgG index, and one or more oligoclonal bands on electrophoresis. ${ }^{27}$ These findings suggest that lymphocytes have migrated to the CNS and synthesise antibodies intrathecally. Furthermore, Sanders et al found evidence of intrathecal activation of the terminal pathway of complement when they examined CSF from patients with primary SS and CNS involvement. ${ }^{28}$

\section{"CNS disease in Siögren's syn- drome may be immunologically mediated"}

Histopathological examination of brain tissue from some patients with CNS-SS has demonstrated a small vessel mononuclear inflammatory and ischaemic/haemorrhagic vasculopathy. ${ }^{29}$ In some anti-Ro positive patients frank necrotising vasculitis (angiitis) was noted. Furthermore, up to $75 \%$ of patients with SS and active CNS disease have been shown to have concomitant active peripheral vasculitis affecting the skin, muscles, and nerves. ${ }^{21}$ Findings compatible with small vessel cerebral angiiitis were also found in a significant proportion of patients with CNS-SS undergoing cerebral angiography. ${ }^{30}$ In these patients there was a strong correlation between abnormal angiography findings and anti-Ro antibody positivity.

Hence, several lines of evidence point towards a small vessel vasculitis being the underlying pathogenic process in at least a proportion of patients with CNS-SS. 
Table 1 CNS involvement in SS: reported prevalence

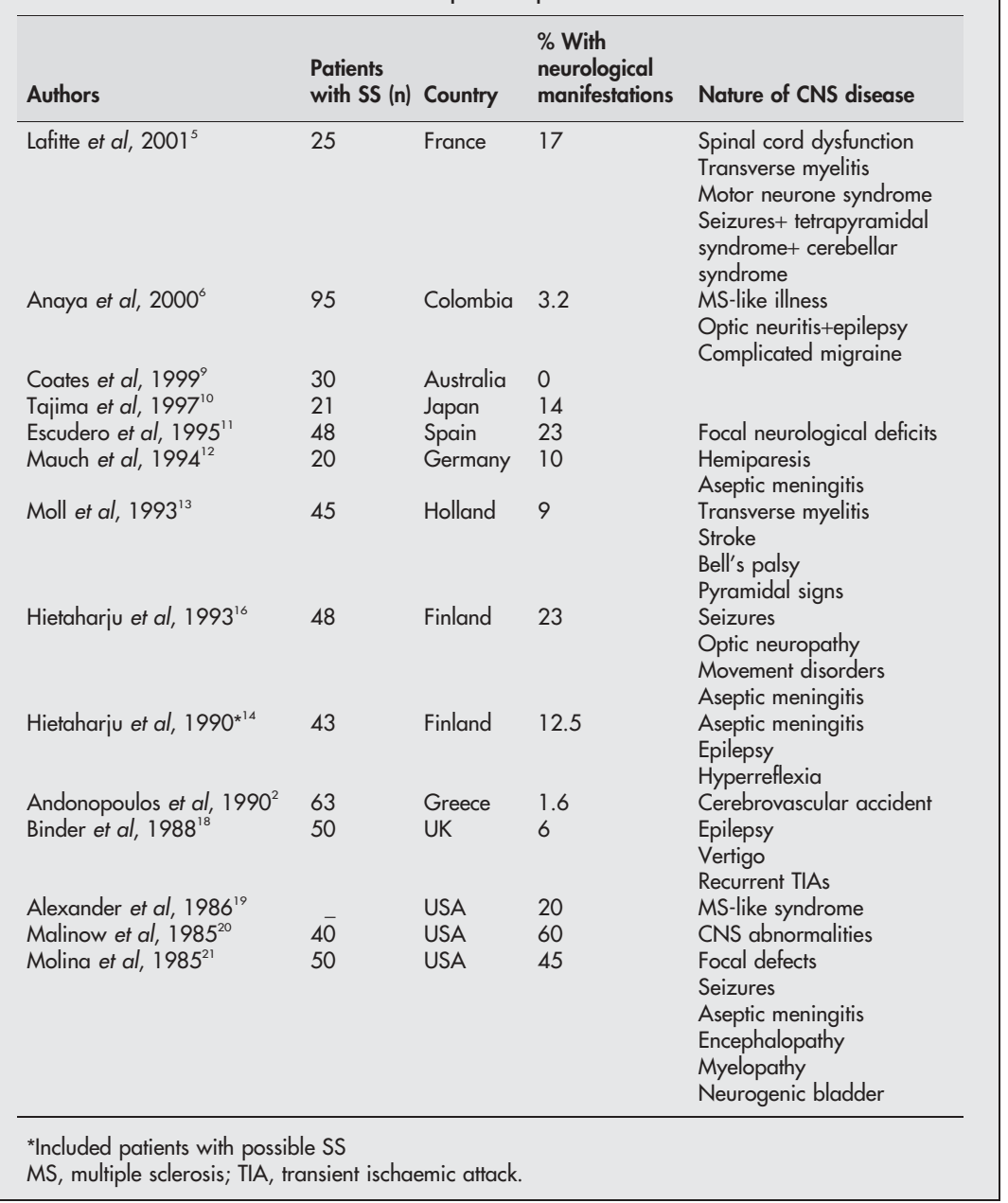

Additional evidence for the potential role of anti-Ro antibodies in the pathogenesis of CNS vasculitis in SS comes from in vitro studies. ${ }^{30}$ Serum samples containing anti-Ro antibodies from patients with CNS-SS were shown to stain the cytoplasm and cell membranes of cultured umbilical vein endothelial

Table 2 Psychiatric involvement in SS: reported prevalence

\begin{tabular}{|c|c|c|c|c|}
\hline Authors & $\begin{array}{l}\text { Patients } \\
\text { with SS (n) }\end{array}$ & Country & $\begin{array}{l}\text { \% With } \\
\text { psychiatric } \\
\text { manifestations }\end{array}$ & $\begin{array}{l}\text { Nature of psychiatric } \\
\text { disease }\end{array}$ \\
\hline Lafitte et al, $2001^{5}$ & 25 & France & 22 & Cognitive dysfunction \\
\hline Belin et al, $1999^{7}$ & 14 & France & 100 & Neuropsychological \\
\hline Govoni et al, $1999^{\circ}$ & 87 & Italy & 8 & Non-focal dysfunction \\
\hline Escudero et al, $1995^{11}$ & 48 & Spain & 29 & Neuropsychiatric \\
\hline Mauch et al, $1994^{12}$ & 20 & Germany & 70 & Cognitive impairment \\
\hline Moll et al, $1993^{13}$ & 45 & Holland & 7 & Psychiatric disorders \\
\hline Spezialetti, et al $1993 t^{15 *}$ & 165 & USA & 80 & $\begin{array}{l}\text { Psychiatric symptoms } \\
\text { Cognitive impairment }\end{array}$ \\
\hline Hietaharju et al, $1990 t^{14}$ & 43 & Finland & 77 & $\begin{array}{l}\text { Psychiatric symptoms- } \\
\text { depression most common }\end{array}$ \\
\hline Drosos et al, $1989^{17}$ & 52 & Greece & 52 & $\begin{array}{l}\text { Introverted hostility } \\
\text { Paranoid ideation } \\
\text { Somatisation } \\
\text { Obsessive-compulsive } \\
\text { behaviour }\end{array}$ \\
\hline Malinow et al, $1985^{20}$ & 40 & USA & 62 & Psychiatric disease \\
\hline
\end{tabular}

antigens. Thus, anti-Ro antibodies may bind to brain endothelial cells and take part in the inflammatory process that leads to vascular damage.

No association has been found between CNS-SS and other autoantibodies associated with vasculitides such as antineutrophil cytoplasmic antibodies. Moreover, ribosomal $\mathrm{P}$ antibodies, formally associated with cerebral lupus, are not detected in patients with primary SS or CNS-SS. ${ }^{15}$ Anticardiolipin antibodies are reported to occur in 5$14 \%$ of patients with primary SS $^{29} 31$ and seem not to be associated with CNS disease. $^{29}$

Single photon emission computed tomography (SPECT) scanning has also contributed to an understanding of the pathogenesis of CNS-SS. Kao et al performed technetium-99m-hexamethylpropylene-amine-oxime SPECT scanning in 16 patients with primary SS with neuropsychiatric manifestations and normal brain magnetic resonance imaging (MRI) results.32 Eighty one per cent of patients had abnormal findings on SPECT scanning, with the temporal lobes being most commonly affected. These findings suggest that brain perfusion abnormalities may account for some of the clinical manifestations in CNS-SS, which exist even in the absence of permanent brain structural changes.

Johnson et al assessed pituitary and adrenal function in eight patients with primary SS and eight healthy controls. ${ }^{33}$ Seven of eight patients with primary SS expressed feelings of anxiety and three of the eight had evidence of depression. Hypoactivity of the hypothalamic-pituitary-adrenal stress axis was found in patients with primary SS compared with healthy controls. Patients with primary SS were characterised by significantly lower adrenocorticotrophin and cortisol levels, as well as by a lack of response to corticotrophin releasing hormone compared with controls. Therefore, some of the mood disorders in primary SS may be partly explained by hypofunction of the hypothalamic-pituitary-adrenal axis.

\section{CLINICAL SPECTRUM OF CNS DISEASE IN SS}

Patients with SS can present with any neurological or psychiatric manifestation. The astute physician should recognise the pattern of neurological involvement (brain, spinal cord, cranial nerves) and according to the level of lesion, proceed to appropriate evaluation. It should be also noted that certain non-focal symptoms, such as cognitive dysfunction or subtle psychiatric disturbances may not be noticed by the assessing physician. To avoid this, a list of questions relating to neurological and 
Table 3 Spectrum of CNS disease in primary SS

\begin{tabular}{l} 
A. Brain \\
Focal \\
Motor and/or sensory deficit \\
Aphasia/dysarthria \\
Brain stem syndrome \\
Cerebellar syndrome \\
Seizures \\
Migraine \\
Non-focal \\
Encephalopathy \\
Aseptic meningitis \\
Cognitive dysfunction/dementia movement \\
disorder \\
Psychiatric disorders \\
B. Spinal cord \\
Transverse myelitis \\
Chronic progressive myelopathy \\
Neurogenic bladder \\
Lower motor neurone disease \\
Brown-Sequard syndrome \\
C. Other \\
Optic neuritis \\
MS-like syndrome \\
\hline
\end{tabular}

psychiatric symptoms must be routinely asked.

\section{"Doctors would avoid missing subtle psychiatric disturbances by asking patients some routine questions"}

However, apart from questionnaires on SS assessing glandular and extraglandular features such as one that recently proposed, ${ }^{34}$ a specific questionnaire for CNS assessment in SS is not currently available. Nevertheless, we consider that a careful history and meticulous neurological examination should be sufficient.

Tables 1, 2, and 3 summarise the reported CNS manifestations in SS. They can involve the brain and spinal cord as well as cranial nerves. ${ }^{29}$

\section{DIAGNOSTIC EVALUATION OF CNS DISEASE IN SS Electrophysiological studies}

Electroencephalography (EEG) is abnormal in one third of patients with SS with severe progressive CNS disease. Patients with focal deficits may show focal slow wave activity, decreased amplitude, or spikes. In patients with epilepsy the EEG may show seizure discharges, whereas in patients with encephalopathy or dementia it may show diffuse slow wave activity. The EEG is useful in detecting sublinical abnormalities that pre-date the development of clinical manifestations in CNS-SS. However, in patients with obvious CNS manifestations the EEG is of limited value.

Multimodality evoked response (MMER) testing, which measures the integrity of neuronal circuits from the periphery via the cerebral cortex, via the visual, auditory, and peripheral nerve pathways, is useful in detecting early subclinical abnormalities and monitoring the response to treatment. ${ }^{29}$

\section{CSF analysis}

CSF analysis in patients with SS and active CNS disease commonly shows lymphocytosis, a raised IgG index, and the presence of one or more oligoclonal bands. ${ }^{27}$ In patients presenting with acute or subacute encephalopathy, CSF examination is also important to exclude the presence of CNS infection (bacterial or viral). Furthermore, when patients have MS-like symptoms, CSF analysis is an important investigation for the diagnosis or exclusion of MS. Unfortunately, data on the role of cytokines in the spinal fluid of patients with SS are not currently available.

\section{Serology}

Both Ro+ and Ro- patients with SS can develop CNS disease. However, Alexander et al demonstrated an association between anti-Ro positivity and the severity of CNS disease as well as with findings on cerebral angiography suggestive of small vessel angiitis. ${ }^{30}$ Therefore in a patient with known SS who presents with CNS manifestations testing for anti-Ro antibodies is of prognostic rather than diagnostic value. $^{29}$

Although the prevalence of antiphospholipid antibodies in SS varies between studies, ${ }^{29}{ }^{35} 36$ it is important to exclude antiphospholipid syndrome in patients with CNS-SS as the clinical manifestations can be similar in the two syndromes. However, they do not seem to have clinical relevance, because they are not associated with thrombotic events or fetal loss. ${ }^{36}$

MRI

MRI scans are more sensitive than computed tomography scans in detecting anatomical abnormalities in CNSSS. ${ }^{37}$ MRI scans of a large proportion of patients with SS with focal CNS disease exhibit multiple areas of increased signal intensity on $\mathrm{T}_{2}$ weighted images, predominantly in subcortical and periventricular white matter. ${ }^{9378}$ These abnormalities can be due to infarction, ischaemia, oedema, or demyelination. An attempt to correlate MRI findings with neuropathological data has shown that some of the MRI abnormalities can be associated with myelin pallor, dilated perivascular (Virchow-Robin) spaces, periventricular gliosis, arteriosclerosis, and infarction. ${ }^{9}$ The prevalence of MRI abnormalities in patients with SS with non-focal/diffuse CNS disease is less clear. Belin et al studied 19 patients with SS, all of whom had neuropsychological abnormalities mostly frontal lobe syndrome and memory problems. ${ }^{7}$ None of the patients had abnormal brain MRI findings. However, in a study of Alexander et al, five of eight patients with psychiatric or cognitive dysfunction had abnormalities on brain MRI imaging. ${ }^{37}$

\section{"MRI detects focal CNS but not always diffuse CNS disease"}

The prevalence of MRI abnormalities in patients with SS without clinical CNS disease is also unclear. According to Alexander such patients have a very low frequency of abnormal MRI scans. ${ }^{29}$ In contrast, Pierot et al studied 15 patients with SS without clinical evidence of CNS disease and found abnormalities in nine $(60 \%)$ on brain MRI, consisting mainly of punctate areas of high signal in the basal ganglia and the white matter of the basal ganglia. ${ }^{39}$ However, at this point it should be emphasised that the incidence of high signal abnormalities in the MRI scans increases with age, rendering the interpretation of the above findings in an SS population, classically middle aged women, rather difficult. ${ }^{40}$

\section{Cerebral angiography}

Cerebral angiography is performed to exclude other causes of CNS disease such as arteriovenous malformations, congenital aneurysms, and other vascular abnormalities and cerebrovascular disease. Up to $45 \%$ of highly selected patients with SS with active CNS disease have angiographic findings suggestive of small vessel vasculitis, such as stenosis, dilatation, or occlusion of small cerebral blood vessels. ${ }^{29}$

\section{DIFFERENTIAL DIAGNOSIS}

Evidently, the spectrum of neurological manifestations in patients with SS is very wide, rendering the list of differential diagnoses quite long; all the mimickers of demyelinating disease should be considered, including inflammatory (SLE, vasculitis, sarcoidosis, Behçet's disease), infectious (Lyme disease, syphilis, progressive multifocal leucoencephalopathy, HTLV-1 infection, herpes zoster), genetic (lysosomal disorders, adrenoleucodystrophy, mitochondrial disorders), metabolic (vitamin B12 deficiency), neoplastic (CNS lymphoma), and spinal (degenerative and vascular malformations) diseases. ${ }^{41}$ Moreover, other neurological disorders, such as dementia, amyotrophic lateral sclerosis, Parkinson's disease, and dorsal root ganglionitis 
should all be included in the differential diagnosis. ${ }^{42}$

However, MS is the main imitator of CNS-SS disease, rendering the distinction between the two entities quite difficult, even by very experienced clinicians. Features found in common between the two disorders include the presenting clinical features, which tend to involve the spinal cord, brain, and the optic tract, producing several symptoms disseminated in time and space, the hallmark of MS. ${ }^{42} 43$ Theoretically, almost all symptoms found in patients with CNS-SS involvement described above, could be attributed to the concomitant presence of MS. However, when peripheral or cranial nerve involvement occurs, as can happen in patients with SS, the diagnosis of MS is less likely.

Ioannidis et al suggest that the coexistence of SS and MS can occur by chance, because both disorders are relatively common in the general population. ${ }^{4}$ On the other hand, several studies over the years report an incidence of SS in patients with MS higher than the expected from chance. However, the issue is controversial; the figures vary among the different reports, ranging from 2 to $16.6 \%{ }^{44-47}$

CSF analysis shows in both disorders a raised IgG index and the presence of oligoclonal bands, suggesting the presence of intrathecal production of IgG. However, it was previously suggested that the number of oligoclonal bands is useful in distinguishing between the two: CNS-SS disorders tend to have fewer than two and MS more than three. $^{42}$

Detection of autoantibodies, such as antinuclear, anti-Ro, anti-La, and rheumatoid factor, found in patients with SS, seems to be a useful tool for the distinction between the two entities. However, it should be borne in mind that positive antinuclear antibodies are commonly found in patients with MS. In a prospective study of patients with MS a high prevalence of antinuclear antibodies, $22.5 \%$, was detected, ${ }^{48}$ whereas anti-Ro/SSA antibodies were reported in a prevalence of $7 \%$ in another study. ${ }^{49}$

\section{"Distinguishing between multiple sclerosis and CNS-SS is difficult"}

MRI studies also are not helpful in differentiating between the two disorders, because periventricular and subcortical lesions are indinguishably found. Therefore, the differential diagnosis of the above disorders should be based mainly on the patient's history, in order to detect features of underlying autoimmune disease, especially sicca symptoms, which should be carefully elicited as they often go unnoticed by patients.

Abnormal MMER testing is also not very helpful for the distinction between the two syndromes, because in CNS-SS about $50 \%$ of patients have one or more abnormal MMER tests.

Apart from MS, CNS-SS disease should be differentiated from CNS involvement in lupus patients. Although similar neurological features can occur in both disorders, several differentiating points should be considered: the onset of the disease, which tends to be abrupt in lupus and insidious, subtle, waning and waxing in SS; the associated autoimmune featuresfor example, rash, serositis, polyarthritis or nephritis in lupus in contrast with sicca symptoms in SS; the different age range of the patients (younger for lupus and older patients for SS); and the distribution of MRI lesions, with white matter involvement in patients with SS, in contrast with grey matter disease in lupus patients. It has been suggested that analysis of CSF from patients with SS shows the presence of atypical mononuclear cells, probably monocytoid $B$ cells, not seen in patients with lupus. Finally, the autoantibody profile (antiRo, anti-La in patients with SS, or anti$\mathrm{Sm}$ and anti-DNA in lupus) tends to give some clues, even though not absolute, for the underlying diagnosis. ${ }^{42}$

\section{TREATMENT}

Treatment of CNS involvement in SS remains largely empirical, based on anecdotal reports and the experience drawn by treating cerebral SLE.

In their comprehensive review, Govoni et al suggest different therapeutic strategies according to different clinical features. ${ }^{50}$ In cases of stable self limiting disease, no intervention is required. In contrast, when signs of activity or progression are evident (for example MRI, CSF abnormalities), aggressive treatment with corticosteroids initially, and intravenous pulses of cyclophosphamide is warranted. The current regimens used include monthly intravenous pulses of cyclophosphamide for 6 to 12 months with a possibility of extension up to 2 years according to the level of clinical response. However, it should be emphasised that large randomised controlled studies are lacking, and protocols used are mainly empirical. Other immunosuppressive drugs tested with various success include azathioprine, methotrexate, and cyclosporin. ${ }^{42}$

The role of plasmapheresis, possibly through removal of circulating pathogenic antibodies seems to be of benefit for some patients, especially together with concurrent immunosuppressive treatment. However, in this case also, convincing published data are not available. A recent report of Canhao and coworkers, demonstrated a beneficial role of intravenous gammaglobulin in the management of CNS vascultis in patients with SS. However, the cost effectiveness of this modality is not well established..$^{51}$

Dementia related to CNS-SS seems to be reversible after inmmunosuppressive treatment. Finally, treatment with tricyclic antidepressant drugs should be used with caution in patients with depressive symptoms because mucosal dryness might be exacerbated, by their anticholinergic action..$^{50}$

\section{CONCLUSIONS}

In conclusion, despite the progress of our understanding of SS, CNS involvement is still a controversial issue. We hope that in the forthcoming years, the pathophysiology of the disorder will be better delineated, the prevalence of the disorder and its optimal treatment will be determined on the basis of well designed multicentre studies.

Ann Rheum Dis 2004;63:616-620.

doi: 10.1136/ard.2003.019497

\section{Authors' affiliations \\ F C Soliotis, C P Mavragani, \\ H M Moutsopoulos, Department of \\ Pathophysiology, School of Medicine, \\ National University of Athens, Greece}

Correspondence to:

Professor H M Moutsopoulos, Department of Pathophysiology, Medical School, University of Athens, 75 M Asias, 115 27, Athens, Greece: hmoutsop@med.voa.gr

\section{REFERENCES}

1 Moutsopoulos HM. Sjögren's syndrome. In Harrison's principles of internal medicine. 15th ed. New York: McGraw-Hill, 2001:1947-8.

2 Andonopoulos AP, Lagos G, Drosos AA, Moutsopoulos HM. The spectrum of neurological involvement in Sjögren's syndrome. Br J Rheumatol 1990;29:21-3

3 Moutsopoulos HM, Sarmas JH, Talal N. Is central nervous system involvement a systemic manifestation of primary Siögren's syndrome? Rheum Dis Clin North Am 1993;19:909-12.

4 loannidis JPA, Moutsopoulos HM. Sjögren's syndrome: too many associations too limited evidence. The enigmatic example of CNS involvement. Semin Arthritis Rheum 1999;29:1-3.

5 Lafitte C, Amoura Z, Cacoub P, Pradat-Diehl P, Picq C, Salachas F, et al. Neurological complications of primary Siögren's syndrome. J Neurol 2001;248:577-84

6 Anaya J-M, Villa L, Restrepo L, Vargas SA, Molina JF, Mantilla RD, et al. Nervous system involvement in primary Siögren's syndrome. Arthritis Rheum 2000;43(suppl):S303 (abstr 1417).

7 Belin C, Moroni C, Caillat-Vigneron N, Debray M, Baudin M, Dumas JL, et al. Central nervous system involvement in Siögren's syndrome: evidence from neuropsychological testing and HMPAO SPECT. Ann Med Interne (Paris) 1999; 150:598-604. 
8 Govoni M, Bajocchi G, Rizzo N, Tola MR, Caniatti L, Tugnoli V, et al. Neurological involvement in primary Siögren's syndrome: clinical and instrumental evaluation in a cohort of Italian patients. Clin Rheumatol 1999; 18:299-303.

9 Coates T, Slavotinek JP, Rischmueller M, Schultz D, Anderson C, Dellamelva $M$, et al. Cerebral white matter lesions in primary Sjögren's syndrome: a controlled study. J Rheumatol 1999;26:1301-5.

10 Tajima Y, Mito Y, Owada Y, Tsukishima E, Moriwaka F, Tashiro K. Neurological manifestations of primary Siögren's syndrome in Japanese patients. Intern Med 1997;36:690-3.

11 Escudero D, Latorre P, Codina M, Coll-Canti J, Coll J. Central nervous system disease in Sï̈gren's syndrome. Ann Med Interne (Paris) 1995; 146:239-42.

12 Mauch E, Volk C, Kratzsch G, Krapf H, Kornhuber $\mathrm{HH}$, Laufen $\mathrm{H}$, et al. Neurological and neuropsychiatric dysfunction in primary Siögren's syndrome. Acta Neurol Scand 1994;89:31-5.

13 Moll JW, Markusse HM, Piinenburg JJ, Vecht CJ, Henzen-Logmans SC. Antineuronal antibodies in patients with neurologic complications of primary Sjögren's syndrome. Neurology 1993;43:2574-81.

14 Hietaharju A, Yli-Kerttula U, Hakkinen V, Frey $\mathrm{H}$. Nervous system manifestations in Siögren's syndrome. Acta Neurol Scand 1990;81:144-52.

15 Spezialetti R, Bluestein HG, Peter JB, Alexander EL. Neuropsychiatric disease in Siögren's syndrome: anti-ribosomal $\mathrm{P}$ and antineuronal antibodies. Am J Med 1993;95: 153-60.

16 Hietaharju A, Jantti V, Korpela M, Frey $\mathrm{H}$. Nervous system involvement in systemic lupus erythematosus, Sjögren syndrome and scleroderma. Acta Neurol Scand 1993;88:299-308.

17 Drosos AA, Andonopoulos AP, Lagos G Angelopoulos NV, Moutsopoulos HM. Neuropsychiatric abnormalities in primary Siögren's syndrome. Clin Exp Rheumatol 1989:7:207-9

18 Binder A, Snaith ML, Isenberg D. Sjögren's syndrome: a study of its neurological complications. $\mathrm{Br}$ J Rheumatol 1988;27:275-80.

19 Alexander EL, Malinow K, Lejewski JE, Jerdan MS, Provost TT, Alexander GE. Primary Sjögren's syndrome with central nervous system disease mimicking multiple sclerosis. Ann Intern Med 1986; 104:323-30.

20 Malinow KL, Molina R, Gordon B, Selnes OA, Provost TT, Alexander EL. Neuropsychiatric dysfunction in primary Siögren's syndrome. Ann Intern Med 1985;103:344-50.

21 Molina R, Provost T, Alexander EL. Peripheral inflammatory vascular disease in Sjögren's syndrome. Arthritis Rheum 1985;28:1342-7.

22 Perez B, Kraus A, Lopez G, Cifuentes M, AlarconSegovia D. Autoimmune thyroid disease in primary Sïgren's syndrome. Am J Med 1995;99:480-4.
23 Scofield RH. Autoimmune thyroid disease in systemic lupus erythematosus and Sjögren's syndrome. Clin Exp Rheumatol 1996;14:321-30.

24 Sanchez JL, Aguirre C, Arcos-Burgos OM, Jimenez I, Jimenez M, Leon F, Pareja J, et al. Prevalence of multiple sclerosis in Colombia. Rev Neurol 2000;31:1101-3. [In Spanish.]

25 Sundstrom P, Nystrom L, Forsgren L. Incidence (1988-97) and prevalence (1997) of multiple sclerosis in Vasterbotten County in northern Sweden. J Neurol Neurosurg Psychiatry 2003;74:29-32

26 Celius EG, Vandvik B. Multiple sclerosis in Oslo Norway: prevalence on 1 January 1995 and incidence over a 25 -year period. Eur J Neurol 2001:8:463-9.

27 Alexander EL, Lijewski JE, Jerdan MS Alexander GE. Evidence of an immunopathogenic basis for central nervous system disease in primary Sjögren's syndrome. Arthritis Rheum 1986:29:1223-31.

28 Sanders ME, Alexander EL, Koski CL, Frank MM, Joiner KA. Detection of activated terminal complement (C5b-9) in cerebrospinal fluid from patients with central nervous system involvement of primary Sï̈gren's syndrome or systemic lupus erythematosus. J Immunol 1987; 138:2095-9.

29 Alexander EL. Neurologic disease in Sjögren's syndrome: mononuclear inflammatory vasculopathy affecting central/peripheral nervous system and muscle. A clinical review and update of immunopathogenesis. Rheum Dis Clin North Am 1993;19:869-908.

30 Alexander EL, Ranzenbach MR, Kumar A Kozachuk WE, Rosenbaum AE, Patronas N, et al. Anti-Ro (SS-A) autoantibodies in central nervous system disease associated with Sjögren's syndrome (CNS-SS): clinical, neuroimaging, and angiographic correlates. Neurology 1994;44:899-908.

31 Cervera R, Garcia-Carrasco M, Font J, Ramos M, Reverter JC, Munoz FJ, et al. Antiphospholipid antibodies in primary Sï̈gren's syndrome: prevalence and clinical significance in a series of 80 patients. Clin Exp Rheumatol 1997;15:361-5.

$32 \mathrm{Kao} \mathrm{CH}$, Lan JL, ChangLai SP, Chieng PU. Technetium-99m-HMPAO brain SPECT in Siögren's syndrome. J Nucl Med 1998;39:773-7.

33 Johnson EO, Vlachoyiannopoulos PG Skopouli FN, Tzioufas AG, Moutsopoulos HM. Hypofunction of the stress axis in Siögren's syndrome. J Rheumatol 1998;25:1508-14.

34 Bowman SJ. Collaborative research into outcome measures in Sjögren's syndrome. Update on disease assessment. Scand J Rheumatol Suppl 2002; (1 16):23-7.

35 Pennec YL, Magadur G, Jouquan J, Youinou P. Serial measurements of anticardiolipin antibodies in primary Siögren's syndrome. Clin Exp Rheumatol 1991;9:165-7.

36 Jedryka-Goral A, Jagiello P, D'Cruz DP, Maldykowa H, Khamashta MA, Hughes GR, et al. Isotype profile and clinical relevance of anticardiolipin antibodies in Sjögren's syndrome. Ann Rheum Dis 1992;51:889-91.
37 Alexander EL, Beall SS, Gordon B, Selnes OA, Yannakakis GD, Patronas N, et al. Magnetic resonance imaging of cerebral lesions in patients with the Siögren syndrome. Ann Intern Med 1988;108:815-23.

38 Manthorpe R, Manthorpe T, Sjoberg S. Magnetic resonance imaging of the brain in patients with primary Siögren's syndrome. Scand J Rheumatol 1992;21:148-9.

39 Pierot L, Sauve C, Leger JM, Martin N, Koeger AC, Wechsler B, et al. Asymptomatic cerebral involvement in Sjögren's syndrome: MRI findings of 15 cases. Neuroradiology 1993:35:378-80.

40 Awad IA, Spetzler RF, Hodak JA, Awad CA, Carey R. Incidental subcortical lesions identified on magnetic resonance imaging in the elderly. I. Correlation with age and cerebrovascular risk factors. Stroke 1986:17:1084-9.

41 Trojano M, Paolicelli D. The differential diagnosis of multiple sclerosis: classification and clinical features of relapsing and progressive neurological syndromes. Neurol Sci 2001 ;22(suppl 2):S98-102.

42 Alexander $\mathrm{E}$. Central nervous system disease in Sjögren's syndrome. New insights into immunopathogenesis. Rheum Dis Clin North Am 1992; 18:637-72.

43 Tesar JT, McMillan V, Molina R, Armstrong J. Optic neuropathy and central nervous system disease associated with primary Sjögren's syndrome. Am J Med 1992:92:686-92.

44 de Seze J, Devos D, Castelnovo G, Labauge P, Dubucquoi S, Stojkovic T, et al. The prevalence of Siögren syndrome in patients with primary progressive multiple sclerosis. Neurology 2001:57:1359-63.

45 Ellemann K, Krogh E, Arlien-Soeborg P, Halberg P. Siögren's syndrome in patients with multiple sclerosis. Acta Neurol Scand 1991;84:68-9.

46 Miro J, Pena-Sagredo JL, Berciano J, Insua S Leno C, Velarde R. Prevalence of primary Sjögren's syndrome in patients with multiple sclerosis. Ann Neurol 1990;27:582-4.

47 Noseworthy JH, Bass BH, Vandervoort MK Ebers GC, Rice GP, Weinshenker BG, et al. The prevalence of primary Siögren's syndrome in a multiple sclerosis population. Ann Neurol 1989;25:95-8.

48 Collard RC, Koehler RP, Mattson DH. Frequency and significance of antinuclear antibodies in multiple sclerosis. Neurology 1997;49:857-6.

49 de Andres C, Guillem A, Rodriguez-Mahou M Lopez Longo FJ. Frequency and significance of anti-Ro (SS-A) antibodies in multiple sclerosis patients. Acta Neurol Scand 2001;104:83-7.

50 Govoni M, Padovan M, Rizzo N, Trotta F. CNS involvement in primary Sjögren's syndrome: prevalence, clinical aspects, diagnostic assessment and therapeutic approach. CNS Drugs 2001;15:597-607.

51 Canhao H, Fonseca JE, Rosa A. Intravenous immunoglobulin in the treatment of central nervous system vasculitis associated with Sjögren's syndrome. J Rheumatol 2000;27:1 102-3. 